\title{
Fast inversion of Zeeman line profiles using central moments
}

\section{Stokes $V$ moments and determination of vector magnetic fields}

\author{
P. Mein ${ }^{1}$, H. Uitenbroek ${ }^{2}$, N. Mein ${ }^{3}$, V. Bommier ${ }^{1}$, and M. Faurobert ${ }^{4}$ \\ ${ }^{1}$ LESIA, Observatoire de Paris, PSL Research University, CNRS, Sorbonne Universités, UPMC Univ. Paris 06, Univ. Paris Diderot, \\ Sorbonne Paris Cité, 5 place Jules Janssen, 92195 Meudon, France \\ e-mail: Pierre.Mein@obspm. fr \\ 2 National Solar Observatory, Sacramento Peak, PO Box 62, Sunspot, NM 88349, USA \\ 3 Observatoire de Paris, 5 place Jules Janssen, 92195 Meudon, France \\ ${ }^{4}$ UMR 6525 H. Fizeau, Université de Nice Sophia Antipolis, CNRS, Observatoire de la Côte d'Azur, Campus Valrose, 06108 Nice, \\ France
}

Received 30 January 2015 / Accepted 30 March 2016

\begin{abstract}
Context. In the case of unresolved solar structures or stray light contamination, inversion techniques using four Stokes parameters of Zeeman profiles cannot disentangle the combined contributions of magnetic and nonmagnetic areas to the observed Stokes $I$. Aims. In the framework of a two-component model atmosphere with filling factor $f$, we propose an inversion method restricting input data to $Q, U$, and $V$ profiles, thus overcoming ambiguities from stray light and spatial mixing.

Methods. The $V$-moments inversion (VMI) method uses shifts $S_{V}$ derived from moments of $V$-profiles and integrals of $Q^{2}, U^{2}$, and $V^{2}$ to determine the strength $B$ and inclination $\psi$ of a magnetic field vector through least-squares polynomial fits and with very few iterations. Moment calculations are optimized to reduce data noise effects. To specify the model atmosphere of the magnetic component, an additional parameter $\delta$, deduced from the shape of $V$-profiles, is used to interpolate between expansions corresponding to two basic models.

Results. We perform inversions of HINODE SOT/SP data for inclination ranges $0<\psi<60^{\circ}$ and $120<\psi<180^{\circ}$ for the $630.2 \mathrm{~nm}$ Fe I line. A damping coefficient is fitted to take instrumental line broadening into account. We estimate errors from data noise. Magnetic field strengths and inclinations deduced from VMI inversion are compared with results from the inversion codes UNNOFIT and MERLIN.

Conclusions. The VMI inversion method is insensitive to the dependence of Stokes $I$ profiles on the thermodynamic structure in nonmagnetic areas. In the range of $B f$ products larger than $200 \mathrm{G}$, mean field strengths exceed $1000 \mathrm{G}$ and there is not a very significant departure from the UNNOFIT results because of differences between magnetic and nonmagnetic model atmospheres. Further improvements might include additional parameters deduced from the shape of Stokes $V$ profiles and from large sets of 3DMHD simulations, especially for unresolved magnetic flux tubes.
\end{abstract}

Key words. line: profiles - Sun: magnetic fields

\section{Introduction}

Most of the inversion techniques processing Zeeman line profiles derive magnetic field components and thermodynamical parameters from least-squares fits of $I \pm S$ profiles, where $S$ represents the Stokes parameters $Q, U, V$ successively. For weak fields, algorithms using central moments (Semel 1967; Uitenbroek 2003; Criscuoli et al. 2013) can provide measurements of magnetic field components. In a previous paper (Paper I; Mein et al. 2011), we extended the inversion by moment calculations of $I \pm S$ profiles to the general case of any magnetic field strength.

Stray light and unresolved structures. Zeeman line profiles can be disturbed by stray light effects from the atmosphere of the Earth, the telescope, and the spectrometer. To eliminate such effects, it was proposed to take neighbouring pixels or the quietest parts of the data set into account to correct the observed profiles in each solar point (Skumanich \& Lites 1987; Orozco Suarez et al. 2007; Asensio Ramos 2009; Del Toro Iniesta et al. 2010).

If magnetic structures are unresolved, magnetic and nonmagnetic areas may contribute simultaneously to the same pixel. The more usual solution to this problem is to define a filling factor $f$ and to assume that the contributions of the magnetic area to the Stokes parameters are $I f, Q f, U f$, and $V f$, while the contribution of nonmagnetic areas is limited to the nonpolarized contribution $I^{\prime}(1-f)$. The intensities $I$ and $I^{\prime}$ can be different. The ratio between areas of magnetic and nonmagnetic components is $f /(1-f)$.

For unresolved magnetic structures, it is well known that products $B f$ can be measured more easily than $B$ and $f$ measured separately (Bommier et al. 2007). For two-component models with magnetic and nonmagnetic areas, independent measurements of $f$ were proposed (Bommier et al. 2009; Bommier 2011) to deduce the magnetic strength $B$. In the special case of the quiet Sun, methods using two lines simultaneously with different Lande factors were used by Stenflo $(1973,2010)$. Complex three-component models have also been proposed with the MISMA code to take different models and different magnetic strengths into account (Viticchié et al. 2011; Viticchié \& Sánchez Almeida 2011). Very low data noise levels are of course required to obtain accurate results with models that depend on many parameters. 
New inversion code insensitive to nonmagnetic model atmosphere. In this paper, we propose an inversion method based on a two-component model using only the polarized parts of the profiles, independent of intensities $I$ and $I^{\prime}$. Magnetic strength $B$ and inclination $\psi$ are derived from moments of $V$-profiles and ratios of integrals of $Q^{2}, U^{2}$, and $V^{2}$. We do not investigate nearly transverse magnetic fields, but similar methods using $Q$-profiles might be developed for magnetic vector inclinations near $90^{\circ}$. We use moments of $V$-profiles to determine barycenters. These are very different from the moments used in Taylor expansions of absorption coefficients by Mathys \& Stenflo (1987) and Solanki et al. (1987).

We begin with the definition of the observable quantities used in the $V$-moments inversion (VMI). We do not take into account the vector field azimuth $\phi$, which might be deduced independently from Stokes $Q$ and $U$ and magneto-optical effects. In our simulations, at first we assume a given model atmosphere for magnetic areas. Later we propose a procedure to specify unknown models in the case of observations (Sect. 7).

We describe the inversion by deducing $B$ and $\psi$ from two quantities $S_{V}$ and $R_{V}$, defined in Sect. 4 . We derive polynomials necessary to extract $B$ and $\psi$ through a brief iteration process via synthetic spectra computed with the RH radiative transfer code (Uitenbroek 2001, 2003). We extend the method to the case of an unknown underlying solar model for the magnetic component using a third quantity $D_{V}$.

Coefficients of polynomials used in the inversion also depend on the instrumental broadening profile and, possibly, on unresolved transverse gradients of the magnetic field. We determine a broadening coefficient in the particuliar case of HINODE SOT/SP data and investigate expected data noise effects for standard models. We process data and compare results of VMI with maps deduced from inversions with UNNOFIT (Bommier et al. 2007) and the HAO MERLIN code $\left(\mathrm{CSAC}^{1}\right)$.

\section{The $\mathrm{RH}$ radiative transfer code}

As in Paper I, we derive synthetic spectra of the $630.25 \mathrm{~nm}$ line with the RH radiative transfer code (Uitenbroek 2001, 2003), which is based on the multilevel accelerated lambda iteration scheme (Rybicki \& Hummer 1991, 1992) to define the required polynomial coefficients for inversion. In the transfer solution, the coupled equations of statistical equilibrium and radiative transfer were solved for a 23-level, 33-line atomic model of Fe I, including the 630.25 and $630.15 \mathrm{~nm}$ lines. Non-LTE iterations were performed in the polarization free approximation to account for the effect of the splitting of the line profile on the radiative rates (Bruls \& Trujillo Bueno 1996). More details can be found in Paper I.

We employed four different one-dimensional hydrostatic solar atmospheric models in the calculations: FALA, FALC, FALF (Fontenla et al. 1993), and MALTM (Maltby et al. 1986). These models represent a quiet cell interior, the averaged quiet sun, the solar network, and a sunspot umbra, respectively. To compute coefficients for the inversion process, we use synthetic profiles with small wavelength steps. However, to simulate noise effects on real observations (Sect. 10), we use synthetic profiles with the same spectral resolution as SOT/SP spectra (spectral step $2.147 \mathrm{pm}$ ). In all of the cases, we performed computation of moments used in the inversion process after profile interpolations by third degree spline functions.

\footnotetext{
1 http://www.csac.hao.ucar.edu/
}
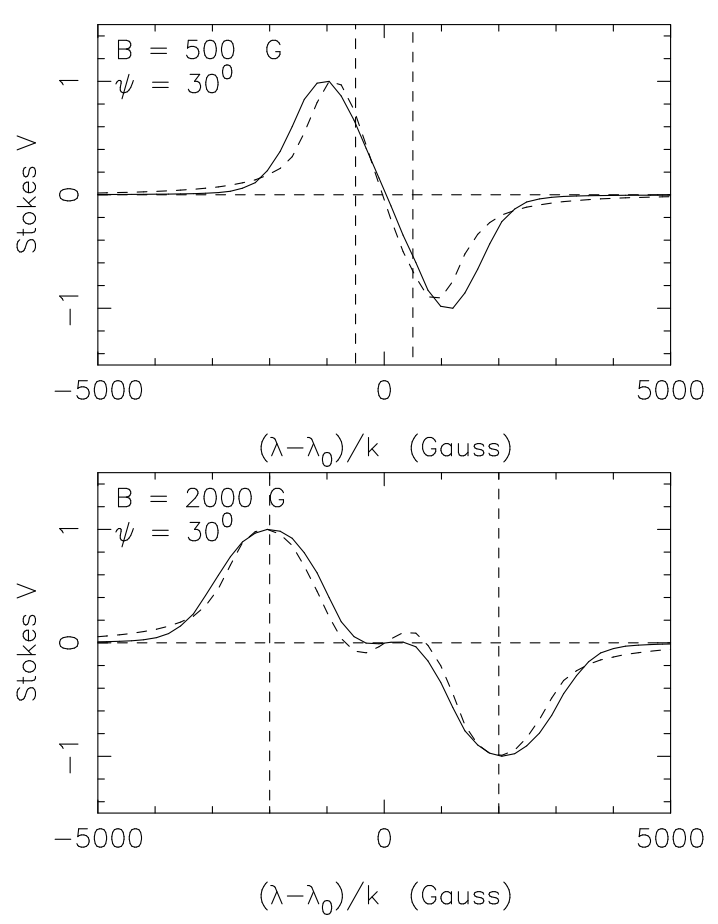

Fig. 1. $V$-profiles for FALC and MALTM models at disk center (full and dashed lines, respectively) with magnetic field strengths $500 \mathrm{G}$ (top) and $2000 \mathrm{G}$ (bottom). Profiles are scaled to their own maximum value.

\section{3. $V$-profiles and vector magnetic field strength $B$}

Figure 1 shows the $V$-profiles of the $630.25 \mathrm{~nm}$ line computed at disk center for FALC and MALTM models with magnetic field strengths $B=500 \mathrm{G}$ and $B=2000 \mathrm{G}$ (inclination $\psi=30^{\circ}$ ).

We denote with $s$ the Zeeman splitting corresponding to the field strength $B$, for an effective Lande factor $\bar{g}$ and a line-center wavelength $\lambda_{0}$ as follows:

$s=k B$

with

$k=4.67 \times 10^{-12} \bar{g} \lambda_{0}^{2}$,

where $s$ and $\lambda_{0}$ are expressed in $\mathrm{nm}$ and $B$ in Gauss.

In both cases, vertical dotted lines are drawn at wavelengths $\lambda=\lambda_{0}+s$ and $\lambda=\lambda_{0}-s$.

As expected, in the stronger field case $(B=2000 \mathrm{G})$, the two lobes of the $V$-profiles approximately coincide at the same positions as the dotted lines. This means that the field strength can be easily deduced from the shifts of the $V$-profiles alone. In the weaker field case $(B=500 \mathrm{G})$, however, the lobes are at larger distances than the dotted lines from line center and the distances depend on the width of the $I$ profile and, thus, on the underlying corresponding atmosphere.

\section{Inversion of $Q, U, V$ profiles: Observable quantities $S_{V}, R_{V}$, and $D_{V}$}

To deduce $B$ and the inclination $\psi$ from Stokes $V, Q$, and $U$ profiles, we propose two observable quantities that are mainly sensitive to $B$ and $\psi$, respectively.

The first quantity, $S_{V}$, is the half-shift between both lobes of the $V^{2}$-profile. We use $V^{2}$ instead of $V$ to reduce the weights of line wings, which are very sensitive to data noise. We checked 
that using $V^{2}$ instead of $V$ significantly increases the accuracy of inversion results.

The second quantity, $R_{V}$, characterizes the ratio between integrals of $Q^{2}+U^{2}$ and $V^{2}+Q^{2}+U^{2}$.

To specify the model atmosphere of magnetic areas inside each solar pixel, we use departures between the half-shift $S_{V}^{\prime}$ of $V$-profiles and the half-shift $S_{V}$ of $V^{2}$-profiles that are sensitive to line wing shapes and noted as $D_{V}$.

We note that $S_{V}, R_{V}$, and $D_{V}$ are very independent of global Doppler shifts of profiles.

\subsection{Shift $S_{V}$ and magnetic field strength $B$}

In the following, moment calculations are performed with profiles interpolated by spline functions leading to a wavelength step divided by four with respect to the original spectral resolution.

Before calculating the half-shift $S_{V}$ between the blue and red lobes of $V^{2}$-profiles, we must determine the central wavelength of the line. To take the possible asymmetry of profiles into account, we separately compute the moments of the positive and negative parts $V_{+}$and $V_{-}$of the $V$-profile. $V_{+}$and $V_{-}$are set to zero where $V$ is negative and positive, respectively,

$\lambda_{0}=\left[\frac{\int_{-\infty}^{+\infty} V_{+}^{2}(\lambda) \lambda \mathrm{d} \lambda}{\int_{-\infty}^{+\infty} V_{+}^{2}(\lambda) \mathrm{d} \lambda}+\frac{\int_{-\infty}^{+\infty} V_{-}^{2}(\lambda) \lambda \mathrm{d} \lambda}{\int_{-\infty}^{+\infty} V_{-}^{2}(\lambda) \mathrm{d} \lambda}\right] / 2$.

We have to compute central wavelengths $\lambda_{b}$ and $\lambda_{r}$ of the blue and red lobes. In the case of observed data, noise may strongly disturb moment calculations of $V^{2}(\lambda)$ because the mean signal is not zero in the far line wings. To avoid this problem, we keep the signal sign and we replace $V^{2}$ by $V \times|V|$ as follows:

$\lambda_{b}=\frac{\int_{-\infty}^{\lambda_{0}} V(\lambda) \times|V(\lambda)| \lambda \mathrm{d} \lambda}{\int_{-\infty}^{\lambda_{0}} V(\lambda) \times|V(\lambda)| \mathrm{d} \lambda}$

$\lambda_{r}=\frac{\int_{\lambda_{0}}^{+\infty} V(\lambda) \times|V(\lambda)| \lambda \mathrm{d} \lambda}{\int_{\lambda_{0}}^{+\infty} V(\lambda) \times|V(\lambda)| \mathrm{d} \lambda}$.

The shift $S_{V}$ is half the wavelength distance between both converted into magnetic field units

$S_{V}=0.5\left(\lambda_{r}-\lambda_{b}\right) / k$

Figures $2 \mathrm{a}$ and $3 \mathrm{a}$ show theoretical values of $S_{V}$ at disk center for different values of $B$ and $\psi$ for the FALC and MALTM models. The line styles depend on the values of $\psi$ : full lines to $30^{\circ}$ and dashed lines to $60^{\circ}$.

We can see that $S_{V}$ depends mainly on $B$ and slightly on $\psi$.

\subsection{Ratio $R_{V}$ and inclination angle $\psi$}

In this section dealing with synthetic spectra, we consider $\psi$-values only between 0 and $90^{\circ}$. The extension to $180^{\circ}$ is easily obtained for observed data, owing to the signs of the $V$-profile lobes.

The ratio of integrated $Q^{2}+U^{2}$ and $Q^{2}+U^{2}+V^{2}$ profiles is 0 for $\psi=0$ and 1 for $\psi=90^{\circ}$. It does not depend on the magnetic vector azimuth and can be used to determine the inclination. The use of the quantity $R_{V}$ seems more convenient for forward polynomial inversion than the unlimited $Q / V$ ratio used, for example, for quiet Sun analysis (Stenflo 2010). (a)

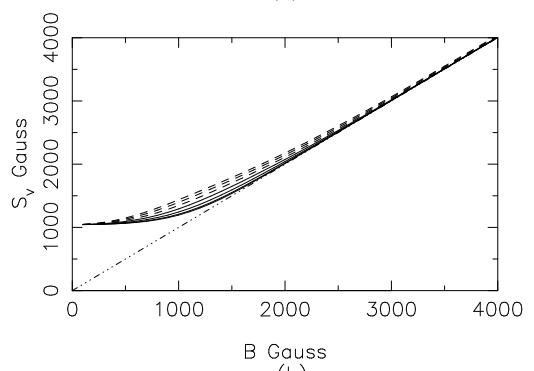

(b)

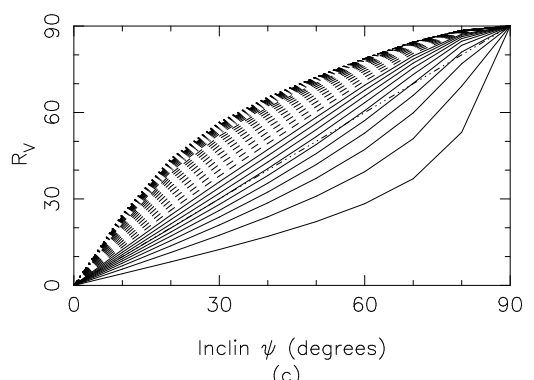

(c)

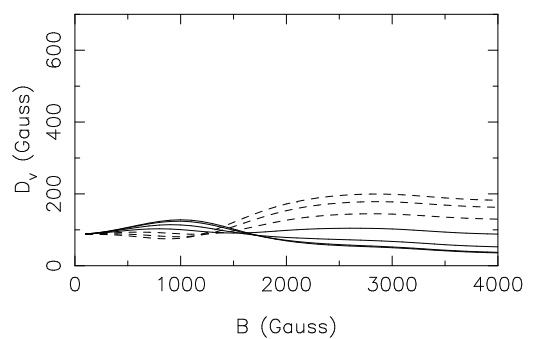

(d)

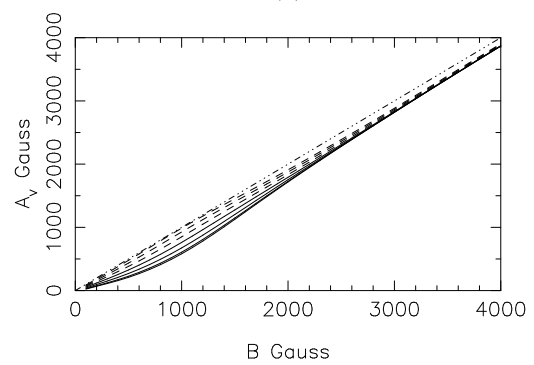

Fig. 2. $S_{V}, R_{V}, D_{V}$, and $A_{V}$ functions for FALC model (Sect. 4).

We define the ratio $R_{V}$ expressed in degrees and characterizing the inclination angle $\psi$ through the equation

$R_{V}=90 \times\left[\frac{\int\left(Q^{2}(\lambda)+U^{2}(\lambda)\right) \mathrm{d} \lambda}{\int\left(V^{2}(\lambda)+Q^{2}(\lambda)+U^{2}(\lambda)\right) \mathrm{d} \lambda}\right]^{1 / 4}$.

Figures $2 \mathrm{~b}$ and $3 \mathrm{~b}$ show theoretical values for FALC and MALTM models. $R_{V}$ increases with $\psi$, regardless of the value of $B$. The line styles depend on the values of $B$ : full lines from $100 \mathrm{G}$ to $1000 \mathrm{G}$, dashed lines from $1100 \mathrm{G}$ to $2000 \mathrm{G}$, dashdotted from $2100 \mathrm{G}$ to $3000 \mathrm{G}$, and dotted from $3100 \mathrm{G}$ to $4000 \mathrm{G}$.

We note that data noise strongly affects values of $R_{V}$ for small $B$ for real observations. If we assume that noise levels are similar for $Q, U$, and $V$, the expected limit of $R_{V}$ for zero magnetic strength is the high value

$R_{V, \lim }=90 \times(2 / 3)^{1 / 4}=81.3^{\circ}$.

To reduce noise effects, we estimate noise in each solar point by the root mean square (RMS) of fluctuations in some wavelength 
(a)

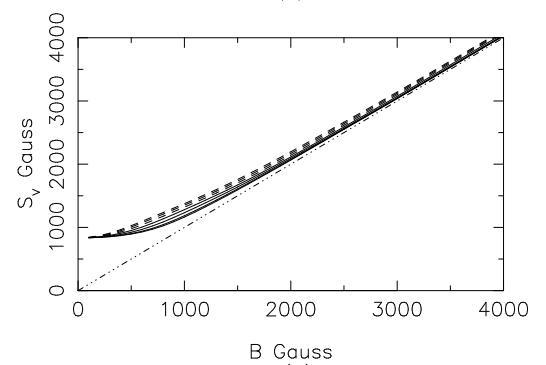

(b)

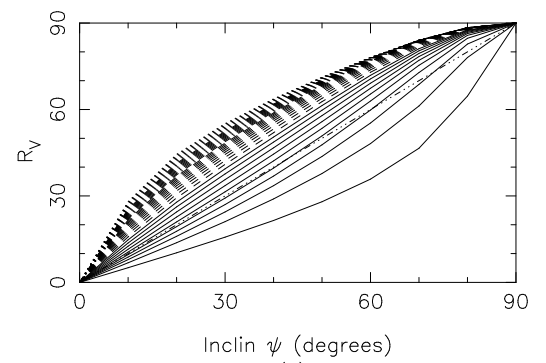

(c)

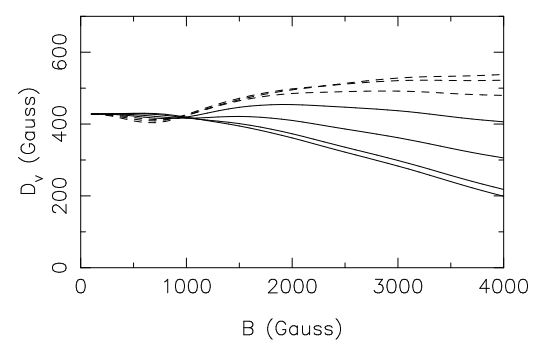

(d)

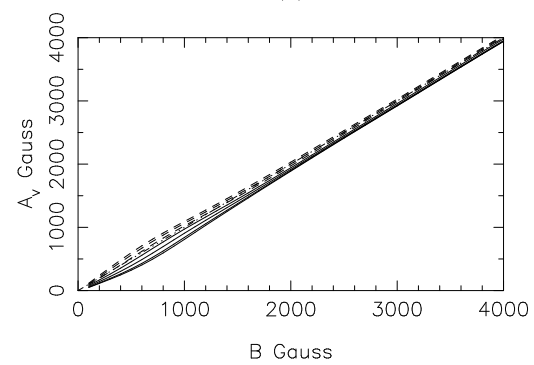

Fig. 3. $S_{V}, A_{V}, R_{V}$, and $A_{V}$ functions for MALTM model.

points at the beginning and end of the available wavelength interval of the line. Then we subtract the result from the corresponding $Q^{2}$ and $U^{2}$ values. We do not modify $V^{2}$ to avoid undetermined $R_{V}$ values. Results are shown in Sect. 10.

\section{3. $S_{V}^{\prime}, D_{V}$, and the model atmosphere}

The difference between shifts $S_{V}^{\prime}$ of $V$-profiles and $S_{V}$ of $V^{2}$-profiles, expressed in Gauss and named $D_{V}$, is used to specify the model atmosphere. The equations defining $S_{V}^{\prime}$ are very similar to Eqs. (4)-(6), i.e.,

$\lambda_{b}^{\prime}=\frac{\int_{-\infty}^{\lambda_{0}} V(\lambda) \lambda \mathrm{d} \lambda}{\int_{-\infty}^{\lambda_{0}} V(\lambda) \mathrm{d} \lambda}$

$\lambda_{r}^{\prime}=\frac{\int_{\lambda_{0}}^{+\infty} V(\lambda) \lambda \mathrm{d} \lambda}{\int_{\lambda_{0}}^{+\infty} V(\lambda) \mathrm{d} \lambda}$

$S_{V}^{\prime}=\left(\lambda_{r}^{\prime}-\lambda_{b}^{\prime}\right) / k$
Figures $2 \mathrm{c}$ and $3 \mathrm{c}$ show theoretical values of

$D_{V}=S_{V}^{\prime}-S_{V}$

The line styles depend on the values of $\psi$ : full lines to $30^{\circ}$, dashed lines to $60^{\circ}$.

\section{5. $A_{V}$, a nearly linear function of $B$ for a given model atmosphere}

The function $S_{V}$ is too far from a linear function of $B$ to be approximated accurately by a polynomial with a small number of nonzero coefficients. If we call $S_{0, M}$ the limit at zero field of the function $S_{v}$ relative to model $M$, we can define a new function,

$A_{V}=\left(S_{V}^{2}-S_{0, M}^{2}\right)^{1 / 2}$,

which is nearly a linear function of $B$. Figures $2 d$ and $3 d$ show plots of $A_{V}$ functions. Line styles are the same as in Fig. 2a.

\section{Polynomials $P_{A}\left(A_{V}, \psi\right)$ and $P_{R}\left(R_{V}, B\right)$ : inversion for a given model atmosphere}

Because $A_{V}$ is a monotonic function of $B$ for all $\psi$ values, it can be inverted by a polynomial least-squares fit, i.e.,

$B=P_{A}\left(A_{V}, \psi\right)=\sum_{i} \sum_{j} a_{i, j} A_{V}^{i-1} \psi^{j-1}$.

In a similar way, $\psi$ can be expressed as a function of $R_{V}$ and $B$ through the equation

$\psi=P_{R}\left(R_{V}, B\right)=\sum_{i} \sum_{j} r_{i, j} R_{V}^{i-1} B^{j-1}$.

The upper values of $i$ and $j$ are typically 7 and 5 for $P_{A}$ and $P_{R}$, respectively.

For a given model atmosphere, the coefficients for polynomials $P_{A}$ and $P_{R}$ can be computed and $B$ and $\psi$ can be recovered from any set of Stokes profiles through a very fast iterative loop,

$B_{n}=P_{A}\left(A_{V}, \psi_{n-1}\right)$

$\psi_{n}=P_{R}\left(R_{V}, B_{n}\right)$

initialized with $B_{0}=0$ and $\psi_{0}=R_{V}$. The number of iteration steps is typically 3 .

We do not discuss the determination of magnetic field azimuth $\phi$, as it is detailed in Paper I; polynomial expansions of magneto-optical effects can be used to deduce directly $\phi$ from $B$, $\psi$, and Stokes $Q$ and $U$.

\section{Polynomials $P_{D}(B, \psi)$ : inversion for an unknown model atmosphere}

For a given model atmosphere, the differences $D_{V}$ can be expanded as functions of $B$ and $\psi$, i.e.,

$D_{V}=P_{D}(B, \psi)=\sum_{i} \sum_{j} d_{i, j} B^{i-1} \psi^{j-1}$.

The upper values of $i$ and $j$ are typically 5 .

We performed simulations for four model atmospheres: FALA, FALC, FALF, and MALTM. They show that $S_{V}, R_{V}$, and $D_{V}$ are very similar for models FALC, FALA, and FALF. Details are given in Sect. (14). As a consequence, the accuracy 
of the inversion process is not degraded by selecting two models, for example, FALC and MALTM, and by interpolating inversion coefficients between both. For any set of $V, Q$, and $U$ profiles, we define an interpolation coefficient $\delta_{V}$ in the following way:

$\delta_{V}=\frac{D_{V}-P_{D, \mathrm{FALC}}(B, \psi)}{P_{D, \mathrm{MALTM}}(B, \psi)-P_{D, \mathrm{FALC}}(B, \psi)}$.

If

$\delta_{V}<-2$

or

$\delta_{V}>3$

the inversion process is given up for the corresponding solar pixel. This may occur for small $B f$ products mainly because of noise. We see later (Figs. 11 and 13) that very few points are involved.

We replace $S_{0, M}$ and polynomials $P_{A}$ and $P_{R}$, defined in Sects. 5 and 6, by

$S_{0, M}=\left(1-\delta_{V}\right) S_{0, \mathrm{FALC}}+\delta_{V} S_{0, \mathrm{MALTM}}$

$P_{A}=\left(1-\delta_{V}\right) P_{A, \mathrm{FALC}}+\delta_{V} P_{A, \mathrm{MALTM}}$

$P_{R}=\left(1-\delta_{V}\right) P_{R, \mathrm{FALC}}+\delta_{V} P_{R, \text { MALTM }}$.

Inside the iteration loop (Eqs. (16), (17)) we introduce Eqs. (18)-(24).

\section{Broadening function for instrumental effects in SOT/SP data}

We analyze SOT/SP data of the active region NOAA10958 observed on 17 May 2007 at 13:01 UT. The SOT/SP instrument (Lites et al. 2013) of the Solar Optical Telescope (Tsuneta et al. 2008) is on board the Hinode mission (Kosugi et al. 2007). Stokes profiles and level 2 outputs from inversions using the HAO "MERLIN" inversion code developed under the Community Spectro-polarimetric Analysis Center are available online ${ }^{2}$.

As in any set of observations, a damping function can be used to mimic instrumental effects and to adjust synthetic profiles to observed data. In the case of unresolved solar structures, an additional effect should be taken into account. Across the same pixel, transverse gradients of the magnetic field may occur and, thereby, broaden Stokes profiles, especially for strong magnetic fields.

Figure 4 shows an example of observed $V$-profile (stars) corresponding to $2500 \mathrm{G}$ (according to UNNOFIT inversion) together with the synthetic MALTM profile (dashed lines). The observed profile is clearly wider than the synthetic profile. It is approximately matched by the synthetic MALTM profile (full line) broadened with the Lorentz function plotted in Fig. 5 and corresponding to the damping coefficient $\gamma=3 \mathrm{pm}$.

To find a unique broadening coefficient that is valid for all data, we turned to the inversion results obtained from UNNOFIT and MERLIN codes. The value $\gamma=3 \mathrm{pm}$ appears to produce a very good agreement in both cases inside a wide magnetic field range corresponding to filling factors close to 1 , as we show in Figs. 14 and 17. All further calculations use that damping coefficient.

\footnotetext{
2 http://WWW.csac.hao.ucar.edu/
}

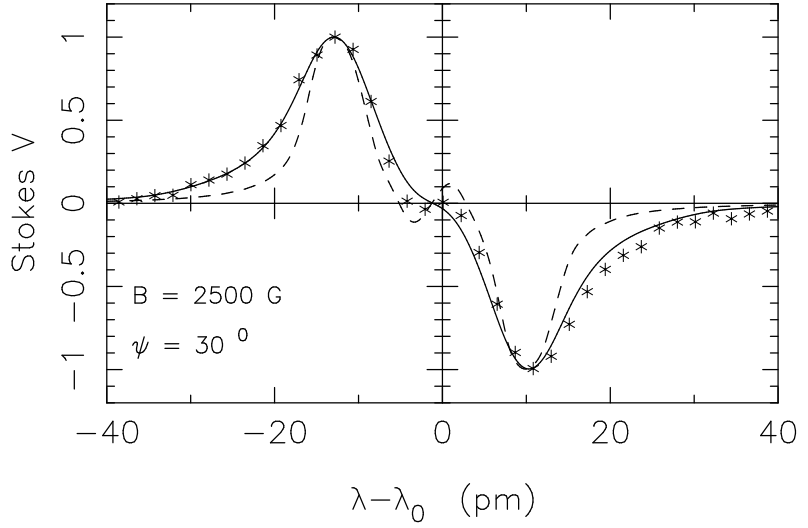

Fig. 4. $V$-profile from SOT/SP data (stars) for magnetic field strengh 2500 G. Normalized synthetic profiles are plotted for MALTM with (full lines) and without (dashed lines) additional broadening.

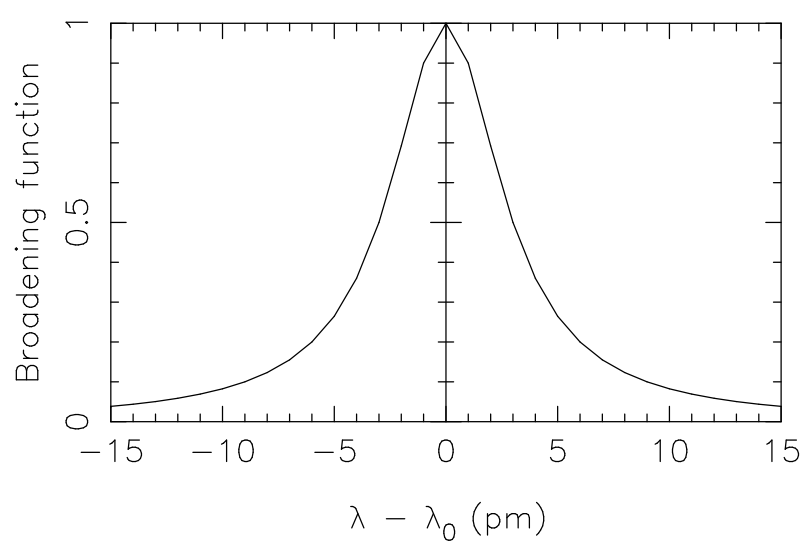

Fig. 5. Lorentz broadening function for synthetic $V$-profiles.

The $\mu$ value is near 0.90 , close to 1 . We can also note that the use of a broadening function automatically adjusts the profile widths to the widths corresponding to the $\mu$ value of observations. So, before broadening, we can use synthetic profiles at disk center.

Using a single coefficient to correct instrumental effects, model fitting, magnetic unresolved fluctuations, and center-tolimb effects is obviously a crude approximation. Further detailed investigations are needed, especially for future data exhibiting even higher resolutions and even lower noise levels. We return to this question in Sect. 15.

\section{VMI inversion and model atmosphere selection}

The synthetic functions $S_{V}, R_{V}, D_{V}$, and $A_{V}$ corresponding to models FALC and MALTM are shown in Figs. 6 and 7.

For a given solar point, the calculation of $A_{V}$ through Eq. (13) is possible only if $S_{V}>S_{0, M}$, where M stands for the model atmosphere that must be selected. We start the iteration by using the $\delta_{V}$ value that corresponds to the model MALTM providing the lowest $S_{0, M}$ value. By starting with the MALTM function we avoid eliminating some points of low magnetic field strengths.

The steps of the inversion are the following:

1) Initialization:

Computation of $S_{V}, R_{V}, D_{V}$

$$
\begin{aligned}
& B_{0}=0 \\
& \psi_{0}=R_{V} \\
& \delta_{V, 0}=1 .
\end{aligned}
$$


(a)

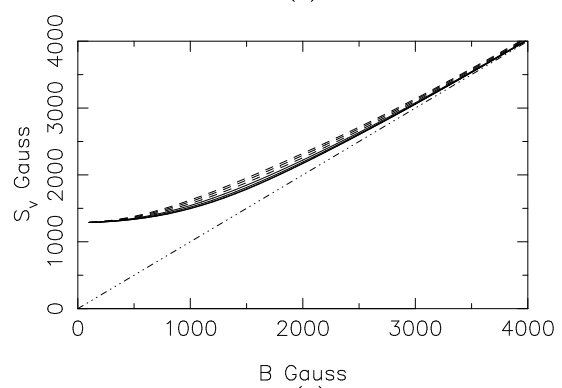

(b)

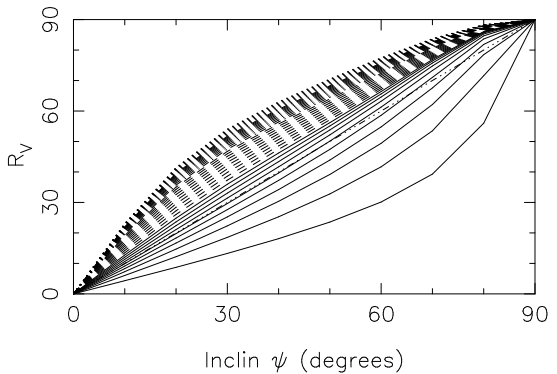

(c)

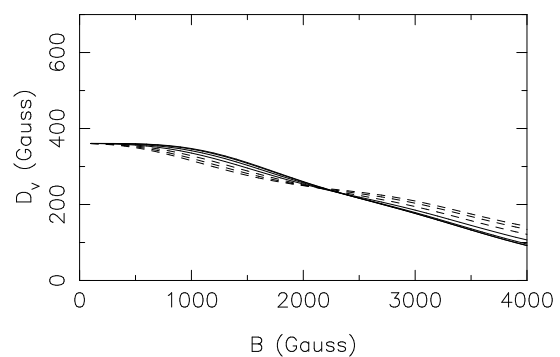

(d)

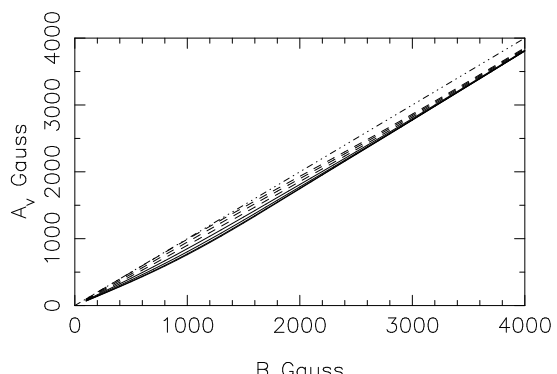

Fig. 6. $S_{V}, A_{V}, D_{V}$, and $A_{V}$ functions for FALC model with additional broadening (Sects. 8, 9).

2) Iteration step $n(n=1,2,3)$ :

Eq. (22) $\rightarrow S_{0, M, n}$

Eq. (13) $\rightarrow A_{V, n}$

Eqs. (18)-(24) $\rightarrow \delta_{V, n}, P_{A, n}, P_{R, n}$

Eqs. (14), (15) $\rightarrow B_{n}, \psi_{n}$

\section{SOT/SP data: Estimates and reduction of noise effects}

\subsection{Data noise level}

As mentioned in Sect. 4.2, we can estimate noise levels in Stokes parameters using RMS values $\epsilon$ over two wavelength intervals: at (a)

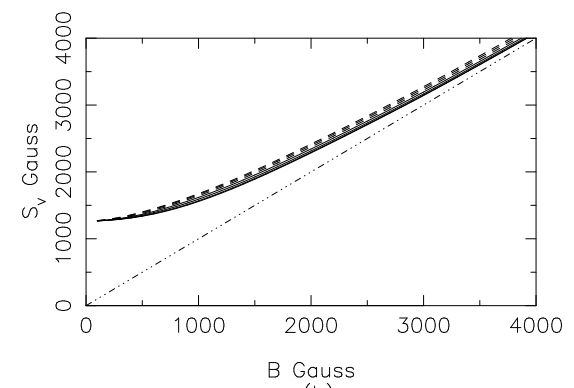

(b)

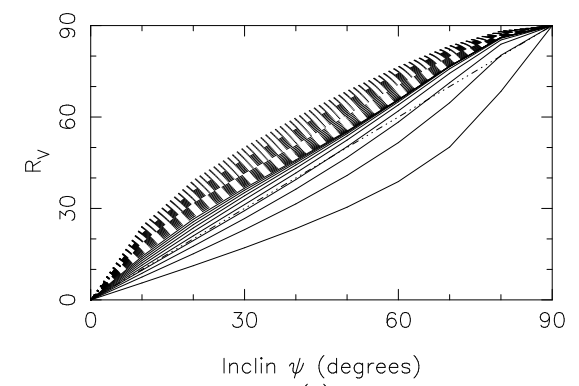

(c)

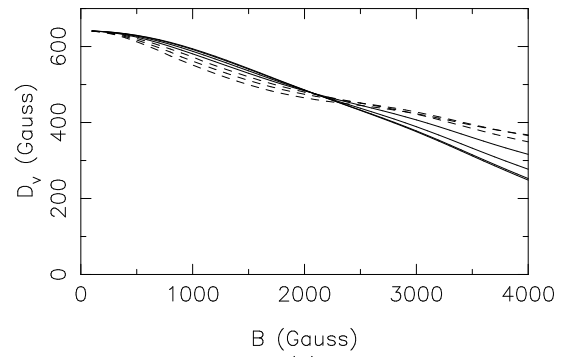

(d)

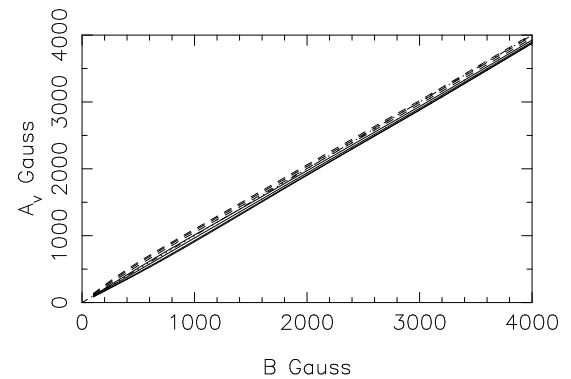

Fig. 7. $S_{V}, A_{V}, R_{V}$, and $D_{V}$ functions for MALTM model with additional broadening.

the beginning and the end of the available spectrum, where $Q, U$, and $V$ are negligible.

Figure 8 shows results obtained across the full set of SOT/SP data with two 5 point intervals corresponding roughly to $-52<\Delta \lambda<-42 \mathrm{pm}$ and $42<\Delta \lambda<52 \mathrm{pm}$, where $\Delta \lambda$ is the wavelength distance from line center. To disentangle results from small and large magnetic fields $B$ or filling factors $f$, we plot the results versus the product $B_{U} f_{U}$ deduced from UNNOFIT inversion. We note that UNNOFIT and MERLIN inversions lead to almost the same results insofar as the product $B f$ is concerned (see Sect. 12, Fig. 16). The values are normalized by the continuum intensity of quiet Sun $I_{Q}$, obtained by averaging continuum intensities over pixels such that $B_{U} f_{U}<20 \mathrm{G}$. Results are presented with circles for $Q$, triangles for $U$, and squares for $V$.

For $B_{U} f_{U}<1000 \mathrm{G}, Q, U$, and $V$ decrease strongly for $|\Delta \lambda|>42 \mathrm{pm}$ and $\epsilon / I_{Q}$ is close to $1.2 \times 10^{-3}$. 


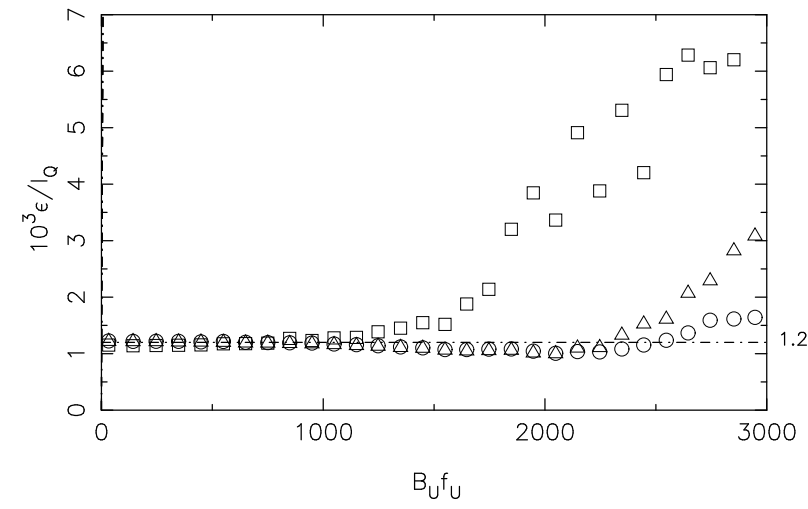

Fig. 8. RMS of Stokes profiles in far wings of the $630.2 \mathrm{~nm}$ line $(|\Delta \lambda|>0.042 \mathrm{~nm})$ in SOT/SP data, divided by the quiet Sun continuum intensity, for noise level determination. Circles for $Q$, triangles for $U$, and squares for $V$.

\subsection{Noise effects reduction for $R_{V}$ computations}

According to Fig. $8, \epsilon / I_{Q}$ remains very near $1.2 \times 10^{-3}$ for $B_{U} f_{U}<2500 \mathrm{G}$ for $Q$ and $U$. This shows that $Q$ and $U$ remain negligible in both 5-point wavelength intervals in a very wide magnetic field range. As mentioned in Sect. 4.2, for each pixel, we subtract from $Q^{2}$ and $U^{2}$ the average values over the two 5-point intervals to reduce noise effects on the $R_{V}$ expression (7). Of course, more accurate results would be obtained with observations including a larger line profile that allows larger intervals.

\subsection{Expected noise effects on VMI inversion}

We use the $1.2 \times 10^{-3} I_{Q}$ noise level to predict effects on VMI inversion results. We can assimilate the continuum intensity to the quiet Sun continuum $I_{Q}$ for synthetic FALC profiles.

However, the ratio between synthetic continuum level intensities of FALC and MALTM is very small, i.e.,

$$
I_{C, \mathrm{MALTM}} / I_{C, \mathrm{FALC}}=0.126
$$

mainly because of temperature differences between both model atmospheres. Moreover, for unresolved structures, we must take into account a geometrical filling factor $f$.

Therefore, to simulate data noise effects, we introduce the stochastic noise at the RMS level of $1.2 \times 10^{-3}$ relative to the continuum intensity in the FALC model in synthetic profiles FALC and MALTM. Because the noise effects depend only on the signal-to-noise ratio, we keep the local continuum (FALC or MALTM) as a reference, but we divide this level by 0.126 for MALTM and by $f$ if the filling factor is not 1 . Of course, different stochastic values are added to quantities $Q, U$, and $V$ for the same point.

We can see expected errors and the RMS of departures in $B$ and $\psi$ in Figs. 9 and 10 for the FALC and MALTM models, with filling factors 1 and 0.1 for FALC, and 1 and 0.7 for MALTM. Triangles mean that owing to noise, $B$ and $\psi$ computations are not always possible in the useful range, mainly because of observed values of $S_{V}$ that are too low.

For inversion of magnetic strengths (upper plots), errors are small in the case of the FALC model and in the MALTM model for $f=1$. They are larger for MALTM model if $f=0.7$ and $B<1400 \mathrm{G}$. In all of the cases, however, errors on $B$ decrease strongly for large magnetic fields. Mean expected $\psi$ errors (lower plots) are computed in the range $500<B<4000 \mathrm{G}$. They are reduced by noise subtraction from $Q^{2}$ and $U^{2}$ integrals (Sect. 4.2).
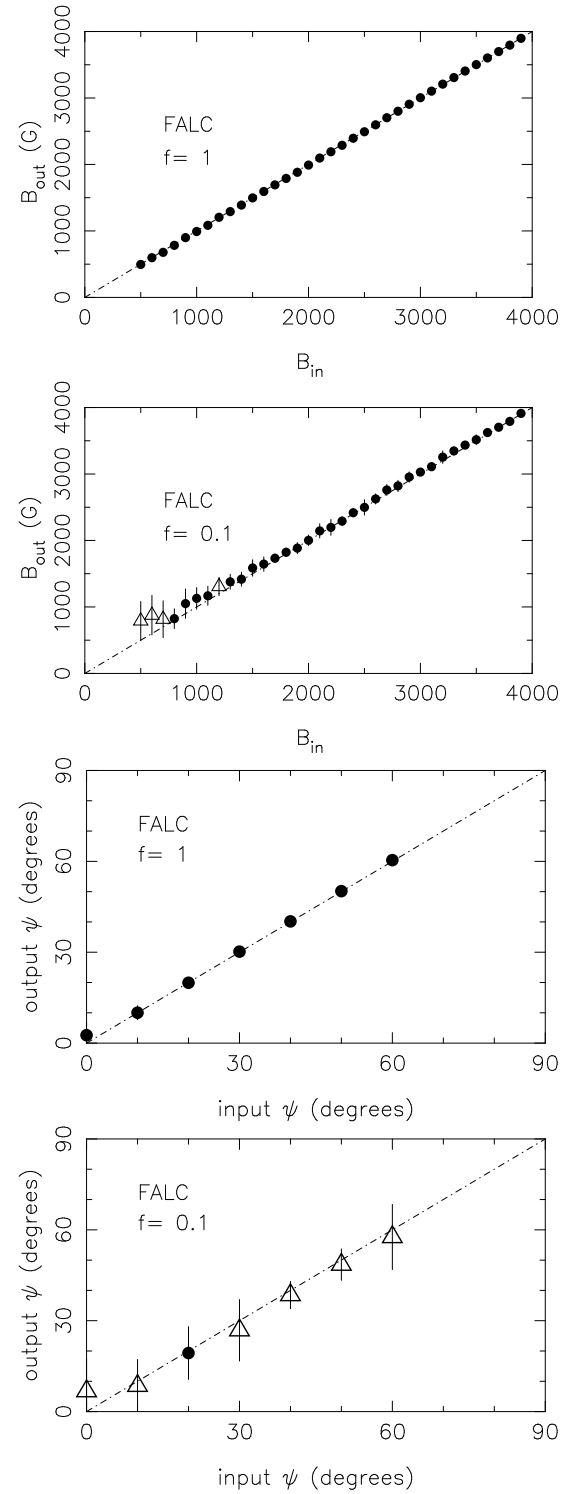

Fig. 9. Inversion of synthetic FALC model data for the case of data noise for filling factors 1 and 0.1 .

\section{Comparison between VMI and UNNOFIT results}

We discuss now the results of VMI inversion of SOT/SP data, notably $B_{V}$ and $\psi_{V}$. We compare them to results of UNNOFIT inversion (Landolfi et al. 1984; Bommier et al. 2007), namely $B_{U}$ and $\psi_{U}$. The range of magnetic strengths is limited by the condition $S_{V}<4500 \mathrm{G}$, corresponding roughly to $B_{V}<4000 \mathrm{G}$. For very large magnetic fields, $V$-profiles cannot be neglected outside the available wavelength range $( \pm 52 \mathrm{pm})$. Additional inversion processes should be developed, for example, using $V$-profile extrapolations or maximum value determinations.

Most of the comparisons are presented versus the product $B_{U} f_{U}$. Indeed, because unresolved structures prevail with similar $B$ values at low magnetic fluxes, $B_{U} f_{U}$ is a good criterion to disentagle ranges of quiet Sun, faculae, and spot penumbrae. Mean values are plotted with a $100 \mathrm{G}$ step in the case of a number of values higher than 10 in each step. Dispersion is materialized by error bars. 

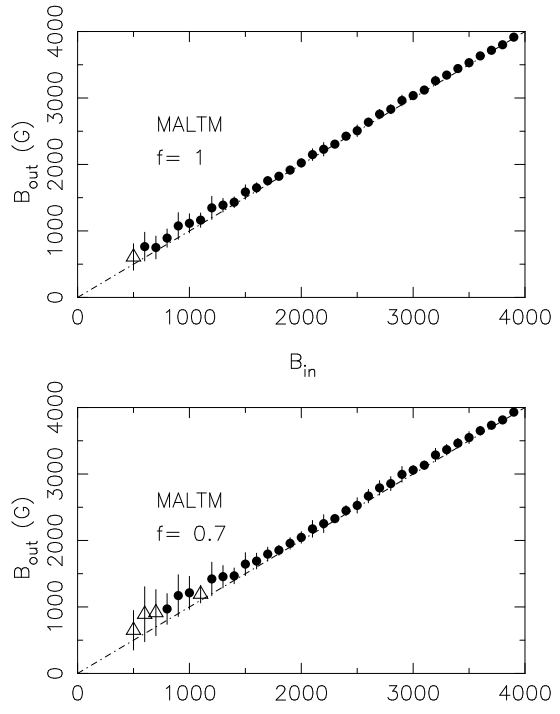

$B_{\text {in }}$
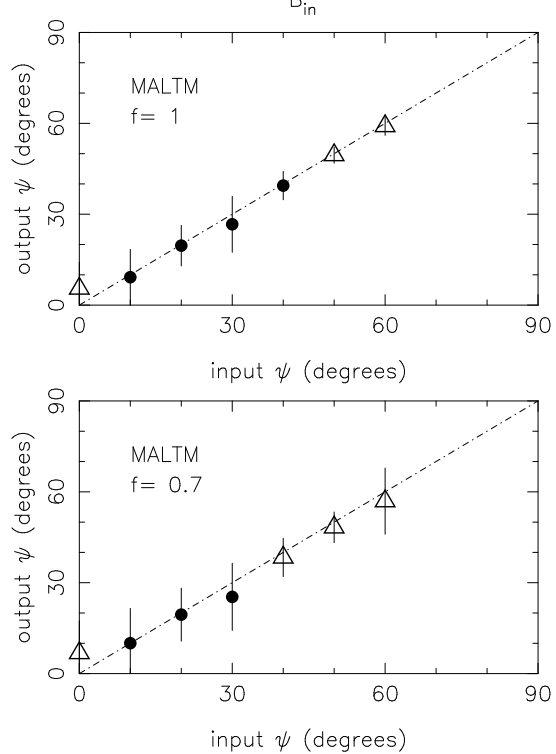

Fig. 10. Inversion of synthetic MALTM model data for the case of data noise for filling factors 1 and 0.7 .

\subsection{Model selection and the $\delta_{V}$ coefficient}

Figure 11 indicates the plot of $\delta_{V}$ versus the product $B_{U} f_{U}$, in the range of inclination angles $0<\psi_{U}<60^{\circ}$ and $120^{\circ}<\psi_{U}<$ $180^{\circ}$. Mean values are black points and the RMS of departures are indicated with vertical lines. Equations (20) and (21) imply $-2<\delta_{V}<3$. The standard deviations, however, are generally smaller than 0.5 , which show that very few points are lost because of this constraint.

We see that, in the range $200<B_{U} f_{U}<1000 \mathrm{G}, \delta_{V}$ is near 0 (FALC), while for higher values it increases up to 1 or more for spot umbrae (MALTM). This shows that the criterion $D_{V}$, which is only the difference between widths of $V$ - and $V^{2}$-profiles, provides an estimate of the model atmosphere selection between FALC and MALTM, and that these two models together match well the whole set of data by representing two extreme cases.

In Fig. 12 we plot the ratio between continuum intensity $I_{C}$ and continuum quiet Sun intensity $I_{Q}$ that is obtained by averaging pixels defined by $B_{U} f_{U}<20 \mathrm{G}$. As expected, this ratio, close to 1 in the range $B_{U} f_{U}<1000 \mathrm{G}$, decreases down to 0.3

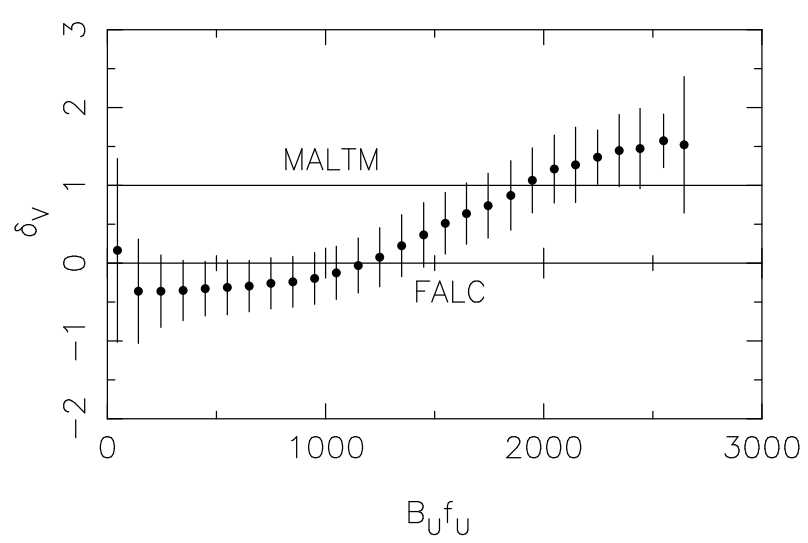

Fig. 11. SOT/SP data: $\delta_{V}$ versus UNNOFIT product $B_{U} f_{U}$.

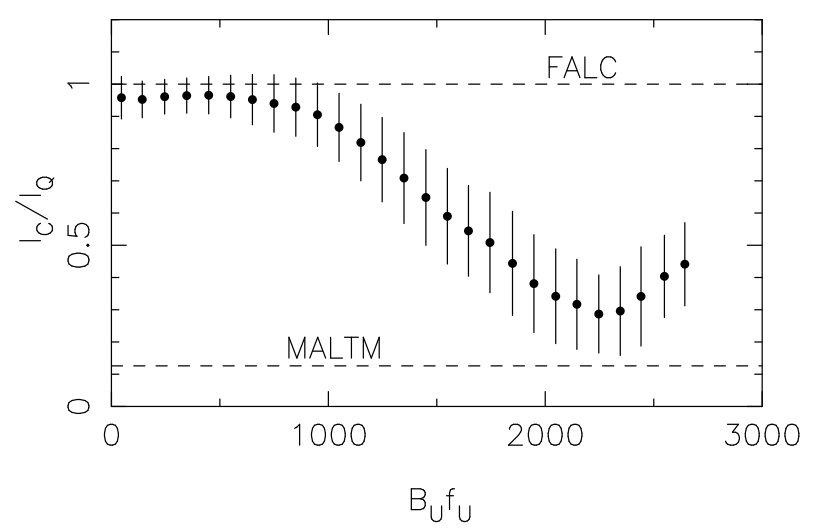

Fig. 12. SOT/SP data: continuum intensity versus UNNOFIT product $B_{U} f_{U}$.

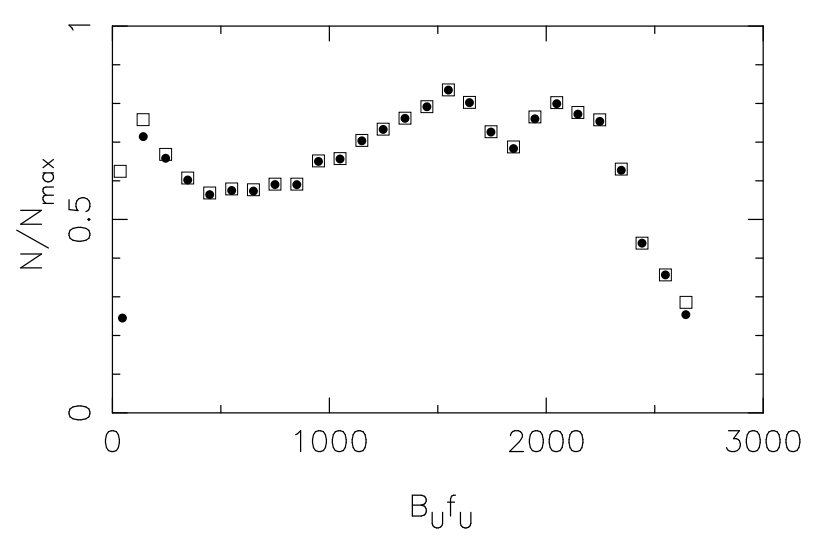

Fig. 13. Numbers of pixels so that $\psi_{U}<60^{\circ}$ or $\psi_{U}>120^{\circ}$ (squares) and numbers of pixels computed by VMI (black points) divided by the total number of available pixels in UNNOFIT inversion.

for $B_{U} f_{U}>2000 \mathrm{G}$. The discrepancy with the expected MALTM value 0.126 may be due to scattered light.

\subsection{Validity range of VMI inversion for SOT/SP data}

Figure 13 shows the number $N$ of available solar pixels divided by the total number of pixels $N_{\max }$ (when $N_{\max }>10$ ) in two different assumptions. Squares correspond to pixels where $\psi_{U}<60^{\circ}$ or $\psi_{U}>120^{\circ}$ and black points to pixels available in VMI inversion. We see that black points are well centered in the corresponding squares, except for very large fields $\left(B_{U} f_{U}>2600 \mathrm{G}\right)$ or points such that $B_{U} f_{U}<200 \mathrm{G}$. 

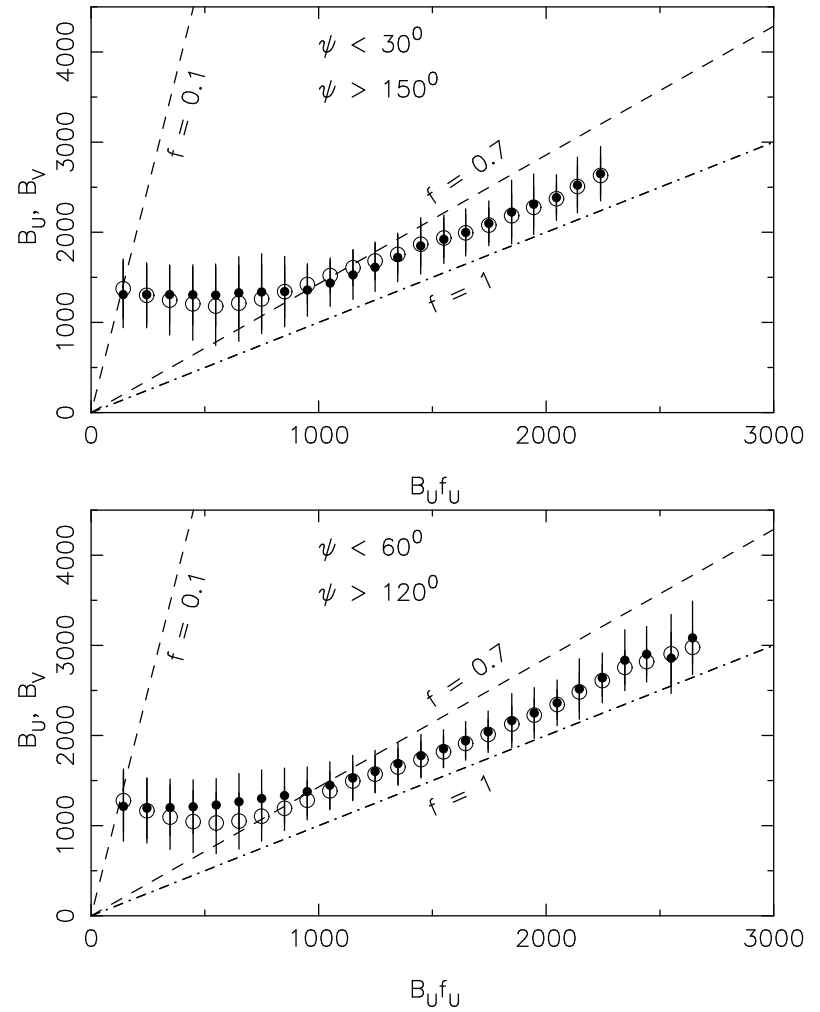

Fig. 14. Magnetic strengths from VMI inversion (black points) and UNNOFIT inversion (circles) in the ranges $0<\psi_{U}<30^{\circ}$ and $150^{\circ}<\psi_{U}<$ $180^{\circ}$ (top) and $0<\psi_{U}<60^{\circ}$ and $120^{\circ}<\psi_{U}<180^{\circ}$ (bottom).

Below this limit, lost pixels correspond generally, either to effects of noise lowering $S_{V}$ values or to the fact that the assumed atmospheric model of magnetic areas is not valid so that the lowest $S_{V}$ value of the model is higher than the observed value. The relative numbers of lost pixels correspond to the departures between the centers of squares and black points. They are very small for $200<B_{U} f_{U}<300 \mathrm{G}$ and practically zero for $300<B_{U} f_{U}<2600 \mathrm{G}$.

\subsection{Magnetic field strengths $B$}

In Fig. 14, we plot $B_{V}$ (black points) and $B_{U}$ (circles) for $B_{U} f_{U}>100 \mathrm{G}$. Each field strength corresponds to an average value across a $100 \mathrm{G} B_{U} f_{U}$ interval including more than 10 pixels. Vertical lines indicate RMS of departures. The data are restricted to points where $\psi_{U}<30^{\circ}$ or $\psi_{U}>150^{\circ}$ (upper diagram) and $\psi_{U}<60^{\circ}$ or $\psi_{U}>120^{\circ}$ (lower diagram). Dashed lines indicate limits corresponding to filling factors $f_{U}$ equal to $1,0.7$, and 0.1 , corresponding to values used in Sect. 10 to estimate noise effects.

To discuss the validity of VMI results in spite of noise effects, we assume that $f_{U}$ values are near the ratio between magnetic and nonmagnetic areas inside each pixel. We consider three cases:

(a) In the range $200<B_{U} f_{U}<1000 \mathrm{G}$, Fig. 11 shows that magnetic areas are mainly relevant to the FALC model. Because all $B_{V}$ computed values are generally higher than $1000 \mathrm{G}$ with $f>0.1$, results appear to be reliable according to Fig. 9 . We can note that if the noise level was reduced by a factor 2, Fig. 9b would apply to the filling factor $f=0.05$. Then Fig. 14 shows that all values such that $B_{U} f_{U}>100 \mathrm{G}$ would become reliable.

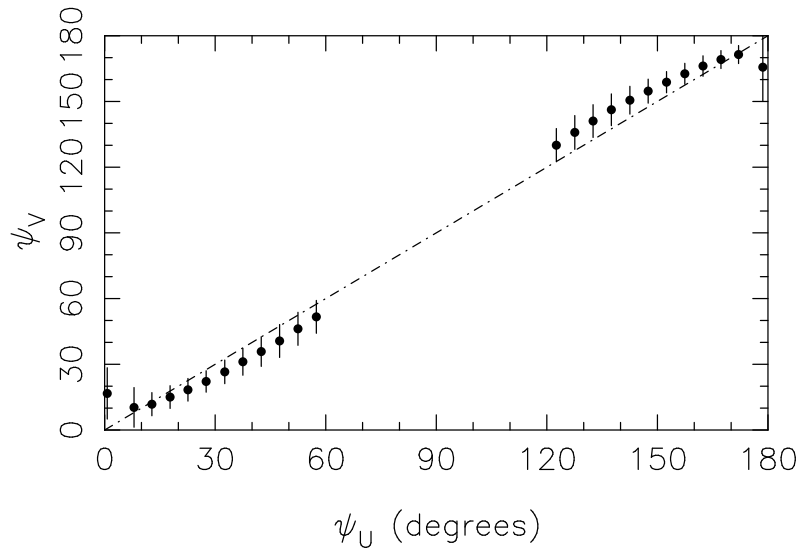

Fig. 15. Inclinations from VMI versus UNNOFIT.

(b) In the range $1000<B_{U} f_{U}<2000 \mathrm{G}$, all $B_{V}$ values correspond approximately to $f>0.7$ with $B_{V}>1000 G$, which indicates reliable results for the FALC model and approximate results for MALTM model, according to Figs. 9 and 10. Anyway, they are in good agreement with $B_{U}$ values.

(c) For higher magnetic fields, some VMI results are a little larger than UNNOFIT results. The magnetic model with only one value of magnetic field is perhaps not relevant to such strong fields. Because VMI uses moments of $V \times|V|$ that are less sensitive to noise than moments of $V$, departures can be due to the different weights assigned to different parts of the profiles. We can expect that magnetic field transverse gradients, which are larger for strong fields than for weak fields, account for observed discrepancies, increasing with magnetic fields. As for the MISMA code, more complex models, including more than two columns or vertical and horizontal gradients and asymmetries, might be investigated.

\subsection{Inclinations $\psi$}

In Fig. 15 we plot inclinations from VMI inversion versus UNNOFIT results in the range $B_{U} f_{U}>200 \mathrm{G}$. As expected, the relative behavior of the results is opposite for angles symmetrical versus $90^{\circ}$. We restrict the discussion to $\psi<90^{\circ}$. We can see from Figs. 6 and 7 that for a given $R_{V}$ value, $\psi$ decreases when $B$ increases. Because VMI $B$ values are a little higher than UNNOFIT values in some ranges, as we show in Fig. 14 (bottom), we may expect $\psi_{V}$ to be somewhat lower than $\psi_{U}$, as it is observed. However, for low $\psi$ values, noise effects still appear to be present in spite of the correction mentioned in Sect. 4.2.

\section{Comparison between VMI and MERLIN results}

\subsection{Magnetic field strengths $B$}

Figure 16 shows that, as expected, $B_{M} f_{M}$ products, extracted from the MERLIN inversion, are very similar to $B_{U} f_{U}$ products used in Sect. 11.

Magnetic field strengths from VMI and MERLIN inversions are plotted in Fig. 17 as functions of $B_{M} f_{M}$ in the ranges $0<$ $\psi_{M}<60^{\circ}$ and $120^{\circ}<\psi_{M}<180^{\circ}$. Three cases can be considered again:

(a) In the range $B_{M} f_{M}<700 \mathrm{G}$, VMI values are higher, especially for very low $B_{M} f_{M}$. Departures are simply because MERLIN code deals with stray light effects, but not with unresolved structures. 


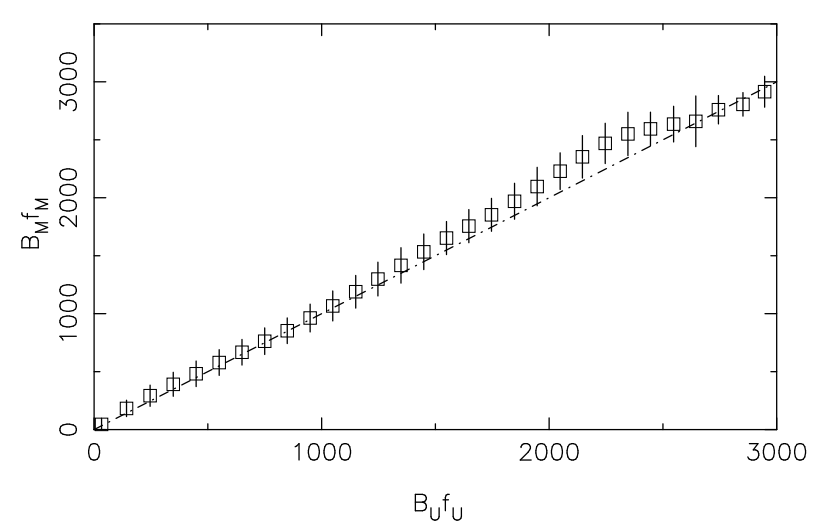

Fig. 16. $B f$ products from MERLIN inversion versus $B f$ products from UNNOFIT inversion.

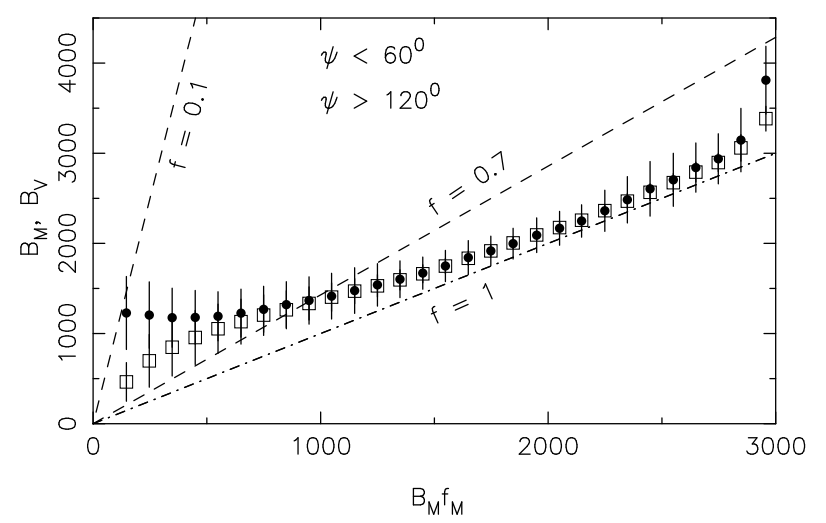

Fig. 17. Magnetic strengths from VMI (black points) and MERLIN inversion (squares) versus $B_{M} f_{M}$ values in the ranges $0<\psi_{M}<60^{\circ}$ and $120^{\circ}<\psi_{M}<180^{\circ}$.

(b) For $700<B_{M} f_{M}<2000 \mathrm{G}$, the agreement is good. It can be noted also that the dispersion of $B$ is very small for each $B_{M} f_{M}$ value.

(c) For $B_{M} f_{M}>2000 \mathrm{G}$, VMI values appear to be a little higher than MERLIN values, especially for very large magnetic fields. As in Sect. 11.3, we can note that unresolved transverse magnetic field gradients, increasing with magnetic strengths, may account for the observed discrepancies.

\subsection{Inclinations $\psi$}

Inclinations are plotted in Fig. 18 in the range $B_{M} f_{M}>200 \mathrm{G}$. They are in rather good agreement with MERLIN results, even for small inclinations.

\section{VMI quick-look without iteration}

As we show in Figs. 6a and 7a, $S_{V}$ values are similar for FALC and MALTM for instrumental broadening, especially for small magnetic strengths. So we can try to replace the interpolation between both basic models by a mean model atmosphere. Moreover, we can use a further simplification by suppressing iterations and replacing curves $A_{V}$ and $R_{V}$ by the first bisector in plots (b) and (d) of Figs. 6 and 7, to get a so-called quick-look VMI ignoring the coupling between $B$ and $\psi$. It can be noted indeed that $R_{V}$ is close to $\psi$ especially for $B$ values near $1000 \mathrm{G}$, that is, in the full range $B_{U} f_{U}<1000 \mathrm{G}$.

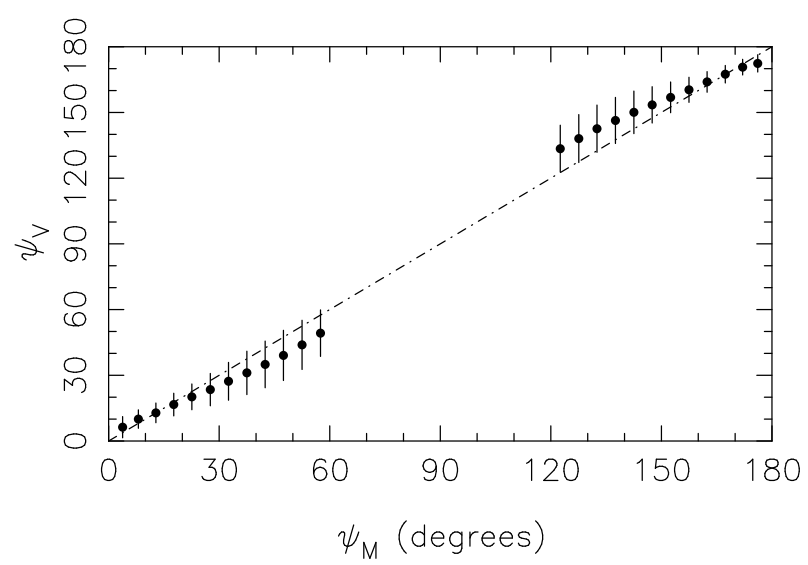

Fig. 18. Inclinations from VMI versus MERLIN inversion code.

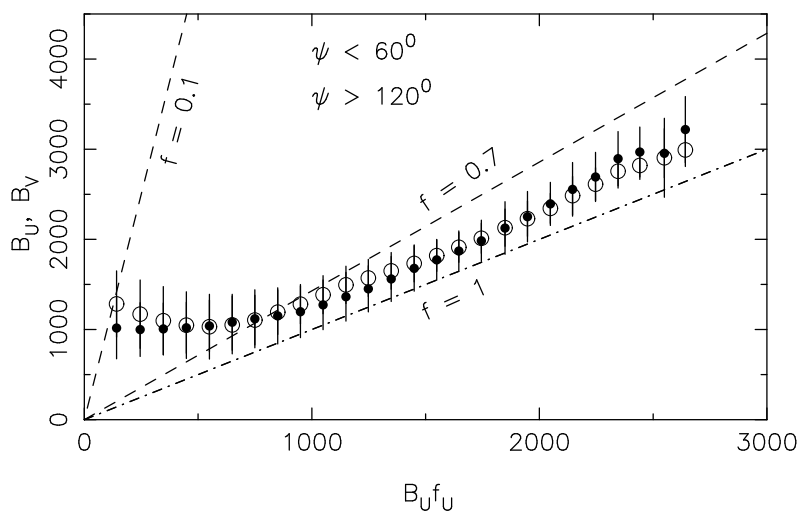

Fig. 19. Same caption as for Fig. 14 (bottom), but for VMI quick-look without iteration. Magnetic strengths from VMI inversion (black points) and UNNOFIT inversion (circles) in the ranges $0<\psi_{U}<60^{\circ}$ and $120^{\circ}<\psi_{U}<180^{\circ}$.

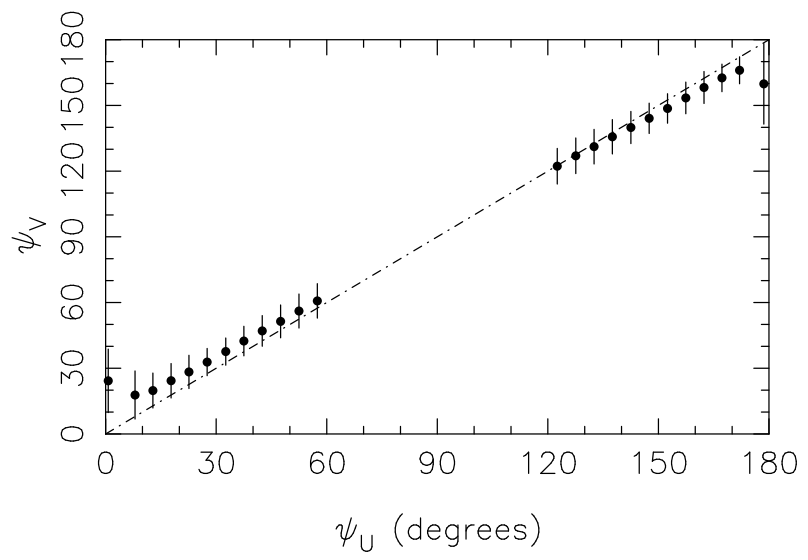

Fig. 20. Same caption as for Fig. 15, but for VMI quick-look without iteration.

Because we know the broadening function and the mean values $S_{V 0}$ of $S_{V}$ for FALC and MALTM near $B=0$, we can reduce the inversion to two equations

$B=A_{V}=\left(S_{V}^{2}-S_{V 0}^{2}\right)^{1 / 2}$

and

$\psi=R_{V}$,

where $S_{V 0}$ is equal to the average between $S_{0, \text { FALC }}$ and $S_{0, \text { MALTM }}$.

Results plotted in Fig. 19 are not very different from results plotted in Fig. 14b in the range $200<B f<2700 \mathrm{G}$. This is a 


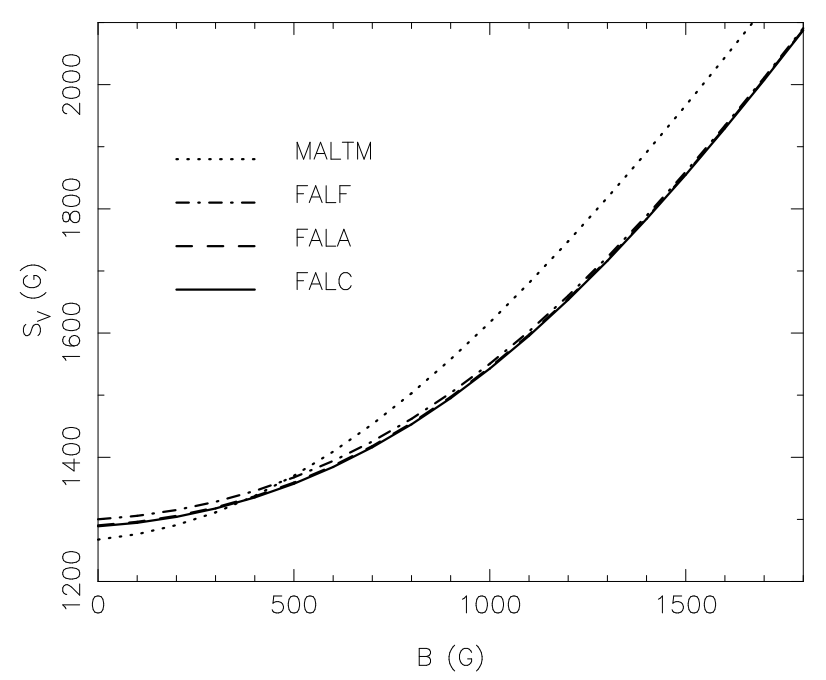

Fig. 21. Synthetic $S_{V}$ values as functions of $B$ for $\psi=0$ and instrumental broadening $\gamma=3 \mathrm{pm}$ for model atmospheres FALC, FALA, FALF, and MALTM. Curves correponding to FALC and FALA are almost superimposed (departures around $2 \mathrm{G}$ ).

clear indication of the small dependency of inversion results on the assumed model atmospheres.

\section{Magnetic field strength dependency on model atmospheres}

\subsection{Synthetic $S_{V}$ and $D_{V}$ for four model atmospheres}

As we show in Figs. 6a and $7 \mathrm{a}, S_{V}$ quantities deviate from $B$ values especially in the weak field regime, so that inversion results are expected to depend more strongly from the assumed model atmospheres in this range. It is, therefore, interesting to estimate the accuracy of the full inversion process using $D_{V}$ quantities and interpolations between model atmospheres.

Figure 21 shows synthetic $S_{V}$ values as functions of $B$ for instrumental broadening $\gamma=3 \mathrm{pm}$, as in Figs. 6a and 7a. Results from model atmospheres FALA and FALF were added to FALC and MALTM. For simplification, the plots are limited to low values of $B$ with $\psi=0$. Similarly, synthetic $D_{V}$ values are plotted in Fig. 22, as in Figs. 6c and 7c.

According to the results from SOT/SP data (Fig. 14), the useful range of $B$ magnetic strength is roughly $B>1000 \mathrm{G}$. In this range, we note that FALC, FALA, and FALF curves are almost superimposed. The $S_{V}$ departures between FALC, FALA, and FALF are always smaller than $10 \mathrm{G}$, while departures between MALTM and FALC (or FALA and FALF) exceed $50 \mathrm{G}$. This shows that the accuracy of inversion results is practically not reduced by eliminating FALA and FALF from the interpolation process between FALC and MALTM.

\subsection{Inversion of SOT/SP data in the range $\mathrm{Bf}>1000 \mathrm{G}$}

For observational data such as the SOT/SP data, magnetic strengths (Fig. 14, top and bottom), depend only slightly on the range of inclination angles. We consider the most simple case of small inclinations $\psi_{U}<30^{\circ}$ and $\psi_{U}>150^{\circ}$, which also takes advantage of a higher signal-to-noise ratio. In Fig. 23, we plot the mean results of SOT/SP data VMI inversion with model interpolation between FALC and MALTM (black points) along with UNNOFIT results (circles), as in the top of Fig. 14. We add by comparison the curves noted $\mathrm{F} \gamma$ (full lines) and $\mathrm{M} \gamma$ (dotted lines)

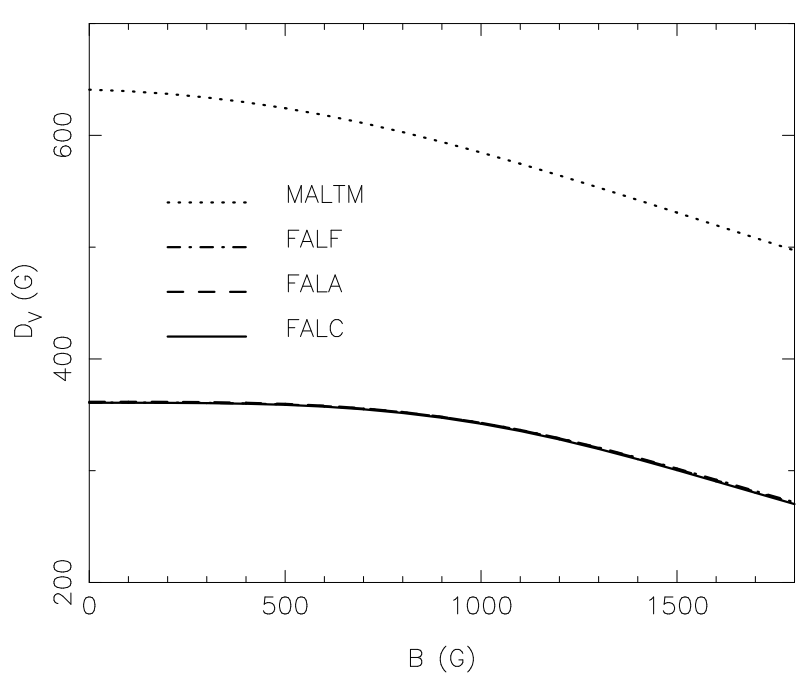

Fig. 22. Synthetic $D_{V}$ values as functions of $B$ for $\psi=0$ and instrumental broadening $\gamma=3 \mathrm{pm}$ for model atmospheres FALC, FALA, FALF, and MALTM. Curves correponding to FALC, FALA and FALF are almost superimposed (departures around $2 \mathrm{G}$ ).

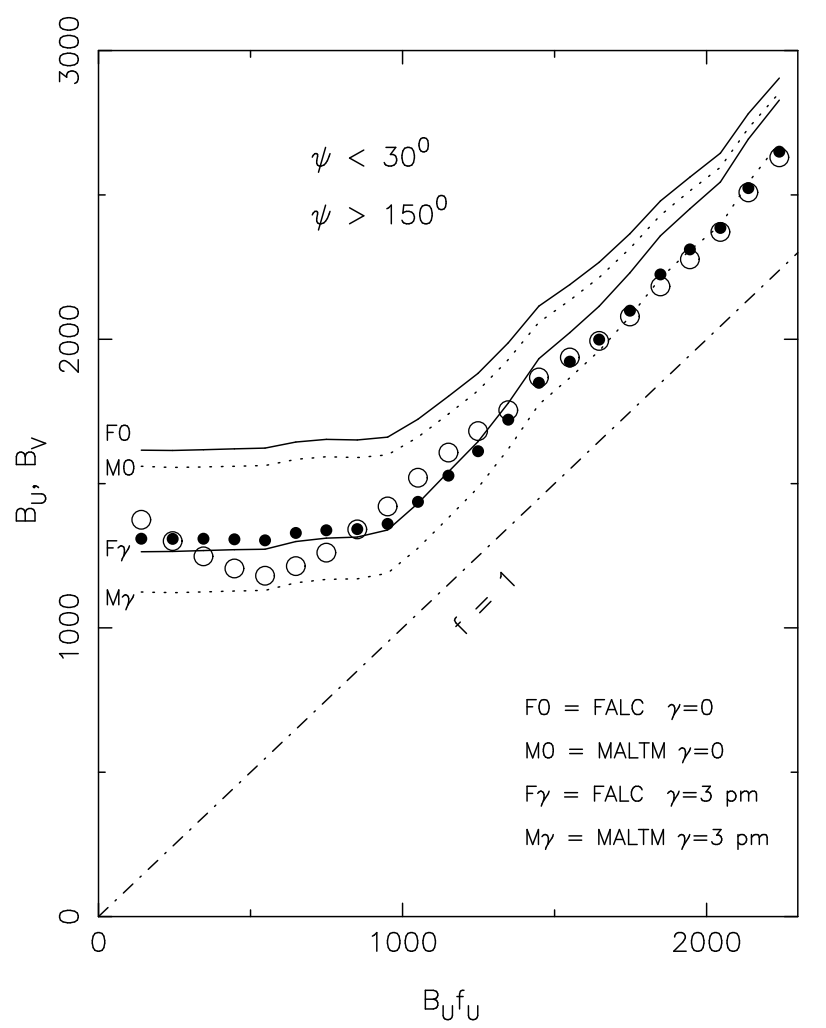

Fig. 23. Magnetic field strength output from UNNOFIT and VMI inversions (circles and black points, respectively). Full and dotted lines correspond to VMI inversions assuming only one given model atmosphere (F0 and M0 without instrumental broadening, $\mathrm{F} \gamma$ and $\mathrm{M} \gamma$ with broadening $\gamma=3 \mathrm{pm})$.

corresponding to results obtained by assuming only one model atmosphere, without interpolations, for FALC and MALTM, with the assumed instrumental broadening $\gamma=3 \mathrm{pm}$.

The VMI and UNNOFIT results behave similarly for $B_{U} f_{U}>1000 \mathrm{G}$. These results leave the $\mathrm{F} \gamma$ line to reach the $\mathrm{M} \gamma$ line, as is expected from the $\delta_{V}$ function plotted in Fig. 11. In the range $B_{U} f_{U}>1300 \mathrm{G}$, the use of a simple linear 
interpolation (Eqs. (19)-(24)) leads to departures less than $50 \mathrm{G}$ between VMI and UNNOFIT.

\subsection{Inversion of SOT/SP data in the range $200<B f<1000 G$}

The agreement is a little less good in the range $B_{U} f_{U}<1000 \mathrm{G}$. According to $\delta_{V}$ values (Fig. 11), FALC is roughly the best model atmosphere, so that results do not depend very much on the interpolation process. Moreover, although filling factors are small (around 0.15 for $B f=200 \mathrm{G}$ ), we expect low data noise effects (even for $f=0.1$, according to Fig. 9). However, we must remind ourselves that for small filling factors, we can expect departures between inversion methods that are using $I$-profiles or not.

It can be noted as well that inversion results using FALC and MALTM separately (curves F $\gamma$ and $\mathrm{M} \gamma$ ) lead to $B$ values that are always higher than $1000 \mathrm{G}$ in the range $B f>200 \mathrm{G}$. According to Fig. 21, they correspond to $S_{V}$ values higher than $1500 \mathrm{G}$ and, therefore, much higher than $S_{0}$ for any of the four models FALC, FALA, FALF, and MALTM. We see also in Fig. 23 that departures between curves $\mathrm{F} \gamma$ and $\mathrm{M} \gamma$ never exceed $150 \mathrm{G}$. This accounts for the relatively small dependency on model atmospheres in this range.

\subsection{Prospects for a better model selection}

To improve the model selection, further investigations are necessary to provide a better understanding of the relationship between thermodynamical parameters and the shape of $V$-profiles. More specifications might be extracted from additional measurements based not only on differences between lobe shifts of $V$ and $V^{2}$-profiles. They would permit the use of nonlinear interpolation between more than one parameter and more than two model atmospheres. The magnetic strength itself might be included in atmosphere specifications during the iteration process. We return to the possibility of new simulations of unresolved magnetic flux tubes through 3D-MHD simulations in Sect. 16.

\section{Magnetic field strength dependency on instrumental broadening}

In Fig. 23 we also plotted the mean results of SOT/SP data inversions without model interpolation for the FALC and MALTM models, but this time without instrumental broadening $(\gamma=0)$. They are noted F0 and M0.

The resulting mean magnetic strengths $B$ are always larger than $1500 \mathrm{G}$. Because values that are too high are obtained in both cases, we assumed that the error was not due to model atmospheres, but mainly to instrumental broadening. The $\gamma$ coefficient is adjusted to get the best agreement with UNNOFIT and MERLIN results especially in the range $B f>1000 \mathrm{G}$, where filling factors are close to 1 and where disturbances due to mixing between magnetic and nonmagnetic spectra do not affect $I$-profiles.

Of course, it would be better in the future to get direct estimates of instrumental effects to check whether the perceived broadening is not in part due to the assumed model atmospheres.

\section{Stokes $V$ amplitude and filling factors}

In the weak field approximation, the $B f$ products and, more generally, the magnetic flux in the line-of-sight direction can be deduced approximately from the maximum Stokes $V$ amplitude by

$B f \cos \psi=0.21 \times 10^{12} \frac{\Delta \lambda_{\mathrm{D}}}{\lambda_{0}^{2} \bar{g}} \frac{|V|_{\max }}{I_{\mathrm{C}}-I_{0}}$,

according to Landi Degl'Innocenti et al. (2004) and Bommier et al. (2009). The parameter $\lambda_{0}$ is the line wavelength in $\mathrm{nm}, \bar{g}$ the effective Lande factor, $\Delta \lambda_{\mathrm{D}}$ the Doppler width, and $I_{\mathrm{C}}-I_{0}$ the difference between continuum and line center intensity in magnetic areas.

For very small filling factors, the observed continuum, line center intensity, and Doppler width correspond to the spectrum of nonmagnetic areas, outside magnetic flux tubes. However, $I_{\mathrm{C}}-I_{0}$ decreases approximately by a factor 8 between the extreme cases of FALC and MALTM (Sect. 10.3), while $\Delta \lambda_{\mathrm{D}}$ decreases by a much smaller factor. Hence, assuming the same model atmosphere inside and outside magnetic areas may lead to errors on determinations of $B f \cos \psi$ and filling factors $f$.

The same problem may also arise in the general case of any magnetic strength for inversions using the same thermodynamical parameters in magnetic and nonmagnetic areas. Further investigations might be able to connect flux tube model atmospheres more accurately with various moments (barycenters and widths) of $V$-profiles. This should lead to more accurate values of filling factors and magnetic fluxes.

Such investigations could be carried out with synthetic polarization profiles obtained from realistic 3D-MHD simulations of the solar photosphere obtained, for example, from the MURaM code (Vögler et al. 2005). These simulations would allow us to estimate and model the effect of unresolved magnetic structures on the observed $V$-profile shapes (see Shelyag et al. 2007). With that prospect, a database giving access to synthetic Stokes spectra of the most commonly used magnetic sensitive lines, computed for various magnetic regimes from 3D-MHD simulations would be a very valuable tool for testing inversion methods.

\section{Conclusions}

VMI specificity and speed. The VMI inversion method can help to make progress in the analysis of unresolved structures by providing magnetic field vectors independently of $I$-profiles. It determines magnetic field vectors for inhomogeneous solar structures in the context of two-component solar models, i.e., magnetic and nonmagnetic. In the same way, results remain reliable if intensity profiles are disturbed by scattered light. The specific point is that VMI does not depend on any nonmagnetic component, since it is independent of the Stokes I profile.

The number of iteration steps typically does not exceed 3 and VMI iterations are very fast. The computing time is less than $3 \times 10^{-4} \mathrm{~s}$ per pixel with a four-processor Xeon computer ( 8 cores, $2.4 \mathrm{GHz}$ ). The useful inclination range is typically $0<\psi<60^{\circ}$ and $120<\psi<180^{\circ}$. It might be complemented by $Q$-moments inversions for $\psi$ angles around $90^{\circ}$.

Comparison with UNNOFIT and MERLIN inversions. SOT/SP data in the $630.2 \mathrm{~nm}$ FeI line have been processed.

Mean VMI $B$ values are always higher than $1000 \mathrm{G}$ and in rather good agreement with UNNOFIT results for $200<B f<$ 2000 G. No really significant departures can be associated with differences between model atmospheres in magnetic and nonmagnetic areas (Sects. 1.2 and 11.3).

Mean VMI values are also in good agreement with MERLIN results for $700<B f<2000 \mathrm{G}$. In the range $B f<700 \mathrm{G}$, 
departures are present because unresolved structures are not included in the MERLIN code.

For very strong fields, VMI $B$ values are slightly higher than those derived with UNNOFIT or MERLIN. Stokes profiles should probably be represented by more complex models with several columns or transverse gradients of magnetic field.

Model atmospheres. We emphasized the importance of model atmosphere selection from the shape of $V$-profiles in Sect. 14. This paper uses the simplest way to accommodate thermodynamical variation, namely with an interpolation between only two model atmospheres, using the difference between lobe shifts in $V$ - and $V^{2}$-profiles. Relationships between thermodynamical parameters and shape of $V$-profiles might be investigated in more detail with new sets of theoretical 3D-MHD simulations

Line broadening and data noise effects: Possible improvements. The accuracy of results should be improved via a direct determination of the $\gamma$ coefficient characterizing instrumental line broadening.

Data noise effects are already reduced in $V$-moments calculations with the use of $V \times|V|$ instead of $V^{2}$ (Sect. 4.1) and with noise subtraction in $Q^{2}$ and $U^{2}$ (Sect. 4.2). Estimates of errors (Figs. 9 and 10), however, show that some reduction of noise level should provide reliable $B$ results for $B f$ values that are much lower than the present limit, which is approximately $200 \mathrm{G}$. A factor 2 should be sufficient to lower the limit down to $100 \mathrm{G}$.

Data noise might also be reduced by longer exposure times on the condition that spatial resolution is not degraded. Wider wavelength intervals across the line profile should also help, not only to increase the accuracy of high magnetic field measurements, but also to allow a better determination and a better correction of noise in the far wings of the line, where Stokes parameters are expected to be negligible.

Profiles of infrared lines, which are more sensitive to low magnetic fields because of larger Zeeman shifts, could also be investigated.
Acknowledgements. We thank the referee for fruitful comments. HINODE is a Japanese mission developed and launched by ISAS/JAXA, with NAOJ as domestic partner and NASA and STFC (UK) as international partners. It is operated by these agencies in cooperation with ESA and NSC (Norway). Hinode SOT/SP Inversions were conducted at NCAR under the framework of the Community Spectro-polarimtetric Analysis Center (CSAC; http://www. csac.hao.ucar. edu).

\section{References}

Asensio Ramos, A. 2009, APJ, 701, 1032

Bommier, V. 2011, A\&A, 530, A51

Bommier, V., Landi Degl'Innocenti, E., Landolfi, M., \& Molodij, G. 2007, A\&A, 464, 323

Bommier, V., Martinez Gonzalez, M., Bianda, M., et al. 2009, A\&A, 506, 1415

Bruls, J. H. M. J., \& Trujillo Bueno, J. 1996, Sol. Phys., 164, 155

Criscuoli, S., Ermolli, I., Uitenbroek, H., \& Giorgi, F. 2013, ApJ, 763, 144

Del Toro Iniesta, J. C., Orozco Suarez, D., \& Bello Rubio, L. R. 2010, ApJ, 711, 312

Fontenla, J. M., Avrett, E. H., \& Loeser, R. 1993, ApJ, 406, 319

Kosugi, T., Matsuzaki, K., Sakao, T., et al. 2007, Sol. Phys., 243, 3

Landi Degl'Innocenti, E., \& Landolfi, M. 2004, Polarization in Spectral Lines (Dordrecht: Kluwer Acad. Publ.)

Landolfi, M., Landi Degl'Innocenti, E., \& Arena, P. 1984, Sol. Phys., 93, 269

Lites, B. W., Akin, D. L., Card, G., et al. 2013, Sol. Phys., 283, 579

Maltby, P., Avrett, E. H., Carlsson, M., et al. 1986, ApJ, 306, 284

Mathys, G., \& Stenflo, J. O. 1987, A\&A, 171, 368

Mein, P., Uitenbroek, H., Mein, N., Bommier, V., \& Faurobert, M. 2011, A\&A, 535, A45

Orozco Suarez, D., Bellot Rubio, L. R., Del Toro Iniesta, J. C., et al. 2007, PASJ, 59, S837

Rybicki, G. B., \& Hummer, D. G. 1991, A\&A, 245, 171

Rybicki, G. B., \& Hummer, D. G. 1992, A\&A, 262, 209

Semel, M. D. 1967, Ann. Astrophys., 30, 513

Shelyag, S., Schüssler, M., Solanki, S. K., \& Vögler, A. 2007, A\&A, 469, 731

Skumanich, A., \& Lites, B. 1987, ApJ, 322, 473

Solanki, S. K., Keller, C., \& Stenflo, J. O. 1987, A\&A, 188, 183

Stenflo, J. O. 1973, Sol. Phys. 32, 41

Stenflo, J. O. 2010, A\&A, 517, A37

Tsuneta, S., Ichimoto, K., Katsukawa, Y., et al. 2008, Sol. Phys., 249, 167

Uitenbroek, H. 2001, ApJ, 557, 389

Uitenbroek, H. 2003, ApJ, 592, 1225

Viticchié, B., \& Sánchez Almeida, J. 2011, A\&A, 530, A14

Viticchié, B., Sánchez Almeida, J., Del Moro, D., \& Berrilli, F. 2011, A\&A, 526, A60

Vögler, A., Shelyag, S., Schüssler, M., et al. 2005, A\&A, 429, 335 